\title{
Correction to: Atlas of Anterior Segment Optical Coherence Tomography
}

\author{
Jorge L. Alió and Jorge L. Alió del Barrio
}

This title was recently Published. The Editors missed to include the acknowledgement text.

Financial Support: This study has been supported by the Red Temática de Investigación Cooperativa en Salud (RETICS), reference number RD16/0008/0012, Funded by Instituto de Salud Carlos III and co-funded by European Regional Development Fund (ERDF), "A way to make Europe".

The updated version of this chapter can be found at https://doi.org/10.1007/978-3-030-53374-8 